\title{
Oxidative stress stimulates $\alpha$-tocopherol transfer protein in human trophoblast tumor cells BeWo
}

\author{
Ronny P. Etzl ${ }^{1,2}$, Thomas Vrekoussis ${ }^{2}$, \\ Christina Kuhn', Sandra Schulze², Johannes M. \\ Pöschl $^{1}$, Antonis Makrigiannakis ${ }^{3}$, Udo Jeschke ${ }^{2, *}$ \\ and Daisy E. Rotzoll ${ }^{1,4}$ \\ ${ }^{1}$ Division of Neonatology, Department of Pediatrics, \\ University of Heidelberg, INF 150, D-69120 Heidelberg, \\ Germany \\ ${ }^{2}$ Department of Obstetrics and Gynaecology, Ludwig \\ Maximilians University of Munich, Maistrasse 11, \\ D-80337 Munich, Germany \\ ${ }^{3}$ Department of Obstetrics and Gynaecology, Medical \\ School, University of Crete, Iraklion, Crete, Greece \\ ${ }^{4}$ Division of Breast, Gynecologic and Perinatal \\ Pathology, Institute of Pathology, University of Leipzig, \\ Liebigstrasse 26, D-04103 Leipzig, Germany
}

\begin{abstract}
$\alpha$-Tocopherol transfer protein $(\alpha$-TTP) has been identified as the major intracellular transport protein for the antioxidant vitamin E ( $\alpha$-tocopherol). Expression of $\alpha$-TTP on the reproductive system has been described both in mouse uterus and lately in the human placenta. The aim of this study was to clarify if placental expression of $\alpha$-TTP can be modified by substances causing oxidative reactions. The human choriocarcinoma cell line BeWo was, therefore, treated with two known pro-oxidants. $\alpha$-TTP expression was determined with immunocytochemistry and evaluated by applying a semiquantitative score. The presence of pro-oxidants in BeWo cells induced $\alpha$-TTP expression. We thus hypothesize that stimulation of $\alpha$-TTP expression by oxidative stress, as this was induced by pro-oxidants, could be part of an antioxidant process occurring in the placenta in the aim of enhancing the supply of $\alpha$-tocopherol. This process could occur both in normal pregnancies, as well as in pregnancy disorders presented with intensified oxidative stress. In that view, this model is proposed for further oxidative stress studies on trophoblast and placenta, on the grounds of clarifying the role of $\alpha$-tocopherol in pregnancy physiology and pathophysiology.
\end{abstract}

\footnotetext{
*Corresponding author:

Prof. Dr. Udo Jeschke

Department of Obstetrics and Gynaecology

Ludwig Maximilians University of Munich

Maistrasse 11

D-80337 Munich

Germany

Tel.: +49-89-51604266

Fax: +49-89-51604916

E-mail: udo.jeschke@med.uni-muenchen.de
}

Keywords: AAPH; $\alpha$-tocopherol transfer protein; BeWo cells; BSO; in vitro model; oxidative stress.

\section{Introduction}

Oxidative stress is the end-result of the imbalance between the reactive oxygen species (ROS) production and the scavenging-antioxidant mechanisms. It can be seen when ROS production outweighs the antioxidant mechanisms' capacity, through which the cell compensates for the damage inflicted by ROS on cell membranes and proteins. Oxidative stress is often present in human physiology. One such case is pregnancy, mainly characterized by increased oxidative metabolism and reduced antioxidant reserve [28]. During early pregnancy, oxidative stress is definitely present as trophoblast cells grow in hypoxic conditions $\left(\mathrm{PO}_{2}<20 \mathrm{~mm} \mathrm{Hg}\right)$ [10]. At the end of the first trimester, placenta vascularization provides an adequate blood supply; as this takes place, further stress has been reported due to a procedure similar to the reoxygenation process $[8,9]$. Moreover, oxidative stress has been shown to reduce trophoblast differentiation/fusion ability, while it increases proliferation and induces angiogenesis [21]. Interestingly, oxidative stress is intensified in pregnancy disorders such as preeclampsia [22], intrauterine growth restriction (IUGR) [27], gestational diabetes mellitus (GDM) [15], and recurrent miscarriages [16]. Several oxidative pathways (mitochondrial electron transport chain, xanthine oxidoreductase, NADPH oxidase, nitric oxide synthase, and heme oxygenase) have been reported in the placenta so far, as well as their compensating antioxidants: manganese superoxide dismutase, copper/zinc superoxide dismutase, catalase, glutathione, glutathione peroxidase, glutathione $S$-transferase, thiol/disulfide oxidoreductase, and vitamins C and $\mathrm{E}$ [21].

Vitamin E ( $\alpha$-tocopherol, vitE) is recognized as a major lipid-soluble, chain-breaking antioxidant located in cellular membranes, protecting polyunsaturated fatty acids against peroxidative damage. Its role in reproductive biology was recognized early in the $20^{\text {th }}$ century $[4,13]$. $\alpha$-Tocopherol transfer protein $(\alpha-\mathrm{TTP})$, with its high affinity for $\alpha$-tocopherol, plays a major role in maintaining adequate plasma $\alpha$-tocopherol levels $[1,13]$. While $\alpha$-TTP was initially described as a cytosolic liver protein [25, 26], it has been identified in pregnant mouse uterus and term human placenta [11, 12, 14]. Recently, it was shown by our group for the first time that $\alpha$-TTP was present in first-trimester placenta [24]. Compared to term placenta, expression of $\alpha$-TTP was reported being reduced in first-trimester syncytiotrophoblast. Major $\alpha$-TTP expression in first-trimester specimens was identified in extravillous 
trophoblast. In addition, expression of lipid peroxidation products such as malondialdehyde and 4-hydroxy-2-nonenal was observed in first-trimester placenta as well, documenting the presence of oxidative processes in early pregnancy.

Despite the above findings, no functional assay has been performed so far, proving that oxidative stress can induce $\alpha$-TTP expression in trophoblast cells. In this study, we proved that oxidative stress can induce $\alpha$-TTP expression on the BeWo human choriocarcinoma cell line - a cell line which generally serves as a model for trophoblast cell assays. Oxidative stress was chemically induced by $2,2^{\prime}$-azobis (2-amidinopropane) dihydrochloride (AAPH - inducing free radicals in the culture medium) [2] and by L-buthionine-( $S, R)$ sulfoximine (BSO - inhibiting de novo synthesis of glutathione) [5]. Considering that this could mimic the corresponding oxidative stress found in human reproduction, we theorized that the current finding could highlight an antioxidant vitE-related mechanism met not only in normal pregnancy, but in pregnancy pathophysiology as well.

\section{Materials and methods}

\section{Cell culture}

BeWo cells were obtained from the European Collection of Cell Culture (ECACC, Salisbury, UK) and cultured in DMEM enriched with $3.7 \mathrm{~g} / \mathrm{L} \mathrm{NaHCO}^{3}, 4.5 \mathrm{~g} / \mathrm{L}$ D-glucose, $1028 \mathrm{~g} / \mathrm{L}$ stable glutamine, and Na-pyruvate (Biochrom AG, Berlin, Germany), and supplemented with $10 \%$ fetal calf serum (PAA Laboratories $\mathrm{GmbH}$, Pasching, Austria), $2.5 \mu \mathrm{g} / \mathrm{mL}$ amphotericin B (Biochrom AG, Berlin, Germany), and $100 \mu \mathrm{g} / \mathrm{mL}$ penicillin/streptomycin (Biochrom AG, Berlin, Germany). Cell culture was maintained in a humidified incubator at $37^{\circ} \mathrm{C}$ with a $5 \% \mathrm{CO}_{2}$ atmosphere.

\section{Treatment of BeWo cells with AAPH and BSO}

BeWo cells $\left(4 \times 10^{5}\right.$ cells per slide) were grown in quadriperms (Greiner Bio-One, Solingen, Germany) in the presence of $0.01 \mathrm{mM}$ AAPH (Polysciences Europe, Eppelheim, Germany) or $0.4 \mathrm{mM}$ BSO (Sigma-Aldrich, Steinheim, Germany), as previously described $[2,5]$. Slides were incubated for 4, 6, 8, 24, and $48 \mathrm{~h}$. Untreated cells, cultured for equal durations, served as controls. All experiments were carried out five times in duplicates. Slides were then washed in phosphate-buffered saline (PBS) (Biochrom AG, Berlin, Germany) twice ( 5 min per wash) and frozen at $-80^{\circ} \mathrm{C}$.

\section{Antibody preparation}

The $\alpha$-TTP-specific rat monoclonal antihuman antibody used in the current study was prepared by Prof. D. Rotzoll (University of Leipzig, Germany) [14]. Specificity of the antibody used in this study was determined by staining liver tissue known as positive control for $\alpha$-TTP and by exchanging the primary antibody with an isotype control antibody [rat immunoglobulin G (IgG)] (Figure 1A).

\section{Immunocytochemistry}

Slides were left to warm up to room temperature. Fixation with acetone $(10 \mathrm{~min})$ followed. The slides were then washed in PBS (1 min) and treated with diluted normal serum $-10 \mathrm{~mL}$ PBS containing $150 \mu \mathrm{L}$ rabbit serum (Vector Laboratories, Burlingame, CA, USA) - for $60 \mathrm{~min}$ at room temperature. Incubation with the monoclonal antibody (dilution 1:200) was then performed at room temperature for $2 \mathrm{~h}$. Immunoreactivity was detected by using the rat IgG VECTASTAIN Elite ABC Kit (Vector Laboratories, Burlingame, CA, USA) according to the manufacturer's protocol. Washes between steps were done using 0.05\% Brij (Sigma-Aldrich, Taufkirchen, Germany) in PBS. Peroxidase staining reaction was done with 3-amino-9-ethylcarbazole (AEC, DakoCytomation, Carpinteria, CA, USA) for $10 \mathrm{~min}$ and stopped with tap water ( $5 \mathrm{~min}$ ). Slides were finally counterstained in Mayer's hematoxylin (30 s) and covered using Aquatex (Merck, Darmstadt, Germany).

The intensity and distribution patterns of the specific immunocytochemical staining were evaluated using a semiquantitative method (immunoreactive score - IRS) as was previously described [23]. Briefly, the IRS score was calculated as the product of the staining intensity ( 0 : no staining; 1 : weak staining; 2 : moderate staining; and 3: strong straining) multiplied by the staining extent ( 0 : no staining; 1: <10\% staining; 2: 11-50\% staining; 3: 51-80\% staining; and 4: $>80 \%$ staining).

\section{Statistical analysis}

The IBM SPSS version 19.0 for Windows was used. IRS was presented in terms of mean \pm SEM (standard error of the mean). Differences between time-points were assessed by Mann-Whitney test. Each observation with $\mathrm{P}<0.05$ was considered significant.

\section{Results}

\section{BeWo cells present a basal $\alpha$-TTP expression}

In the aim of investigating whether there is a basal $\alpha$-TTP expression in BeWo cells, untreated cells were cultured for $4,6,8,24$, and $48 \mathrm{~h}$, serving at the same time as controls to the corresponding experiments with regard to $\alpha$-TTP expression after treatment with BSO and AAPH. A weak to moderate basal expression (Figure 1B), ranging from 3.70 \pm 0.21 to $5.70 \pm 0.66$, was found (Figure $2 \mathrm{C}$ and D). $\alpha$-TTP immunoreactivity was located in the cell cytoplasm. No difference was identified between the different time-points of the study, suggesting that in BeWo cells, $\alpha$-TTP expression is constant despite the BeWo's ability to start fusing to syncytia during the first days in culture.

\section{BSO-induced oxidative stress upregulates $\alpha$-TTP expression in BeWo cells}

A gradual increase in $\alpha$-TTP expression was observed when BeWo cells were treated with $0.04 \mathrm{mM}$ BSO. $\alpha$-TTP expression was minimal after $4 \mathrm{~h}$ of BSO treatment (IRS: $2.90 \pm 0.34$ ), being increased significantly at $6 \mathrm{~h}$ compared to the $4 \mathrm{~h}$ treatment (IRS: $4.00 \pm 0$ vs. $2.90 \pm 0.34$, respectively; $\mathrm{P}=0.023$ ) (Figure 2A). A further significant increase was seen after $8 \mathrm{~h}$ compared to the corresponding $6 \mathrm{~h}$ incubation (IRS: $8.00 \pm 0$ vs. $4.00 \pm 0$, respectively; $\mathrm{P}=0.0001$ ) (Figure $2 \mathrm{~A}$ ). No difference in $\alpha$-TTP expression was seen among 8, 24, and 48 h treatments. Pairwise comparisons between $\alpha$-TTP IRS of BSO-treated cells vs. $\alpha$-TTP IRS of untreated cells revealed that only after $8 \mathrm{~h}$ of treatment with BSO did $\alpha$-TTP expression 


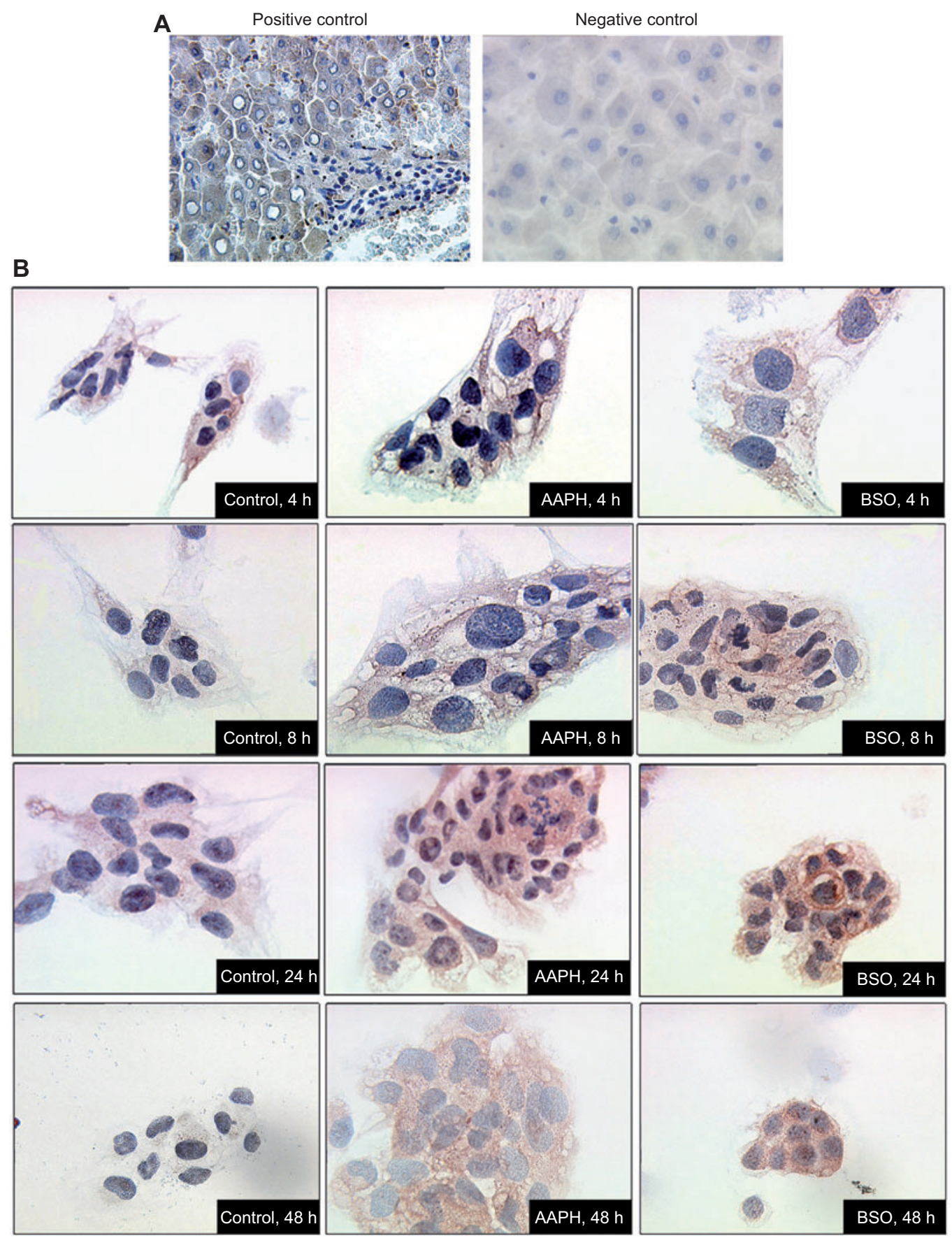

Figure 1 Positive and negative controls for $\alpha$-TTP immunocytochemistry experiments.

(A) Specificity of the antibody used in this study was determined by staining liver tissue known as positive control for $\alpha$-TTP and by exchanging the primary antibody with an isotype control antibody (rat $\operatorname{IgG}$ ). (B) Representative microphotographs presenting $\alpha$-TTP expression in BeWo cells treated with AAPH or BSO. It is clear that both pro-oxidants induce $\alpha$-TTP expression.

differ significantly from the corresponding untreated cells (IRS: $8.00 \pm 0$ vs. $5.70 \pm 0.66$, respectively; $\mathrm{P}=0.023$ ). This significance remained both in the $24 \mathrm{~h}$ treatment (IRS: $8.00 \pm 0 \mathrm{vs}$. $4.30 \pm 0.57$, respectively; $\mathrm{P}=0.0001)$ and in the $48 \mathrm{~h}$ treatment (IRS: $7.60 \pm 0.40$ vs. $3.70 \pm 0.21$, respectively; $\mathrm{P}=0.0005$ ). The current data suggest that BSO-induced oxidative stress causes an upregulation of $\alpha$-TTP expression, this being significant though only at least after an $8 \mathrm{~h}$ effect.

\section{AAPH-induced oxidative stress can also upregulate $\alpha$-TTP expression in BeWo cells}

The time response of $\alpha$-TTP expression in AAPH-treated cells was different than that seen in BSO-treated cells. Up to $8 \mathrm{~h}, \alpha$-TTP expression, although moderate, did not differ significantly between the time-points of the study (Figure 2B). However, after 24 h, $\alpha$-TTP was significantly more expressed 

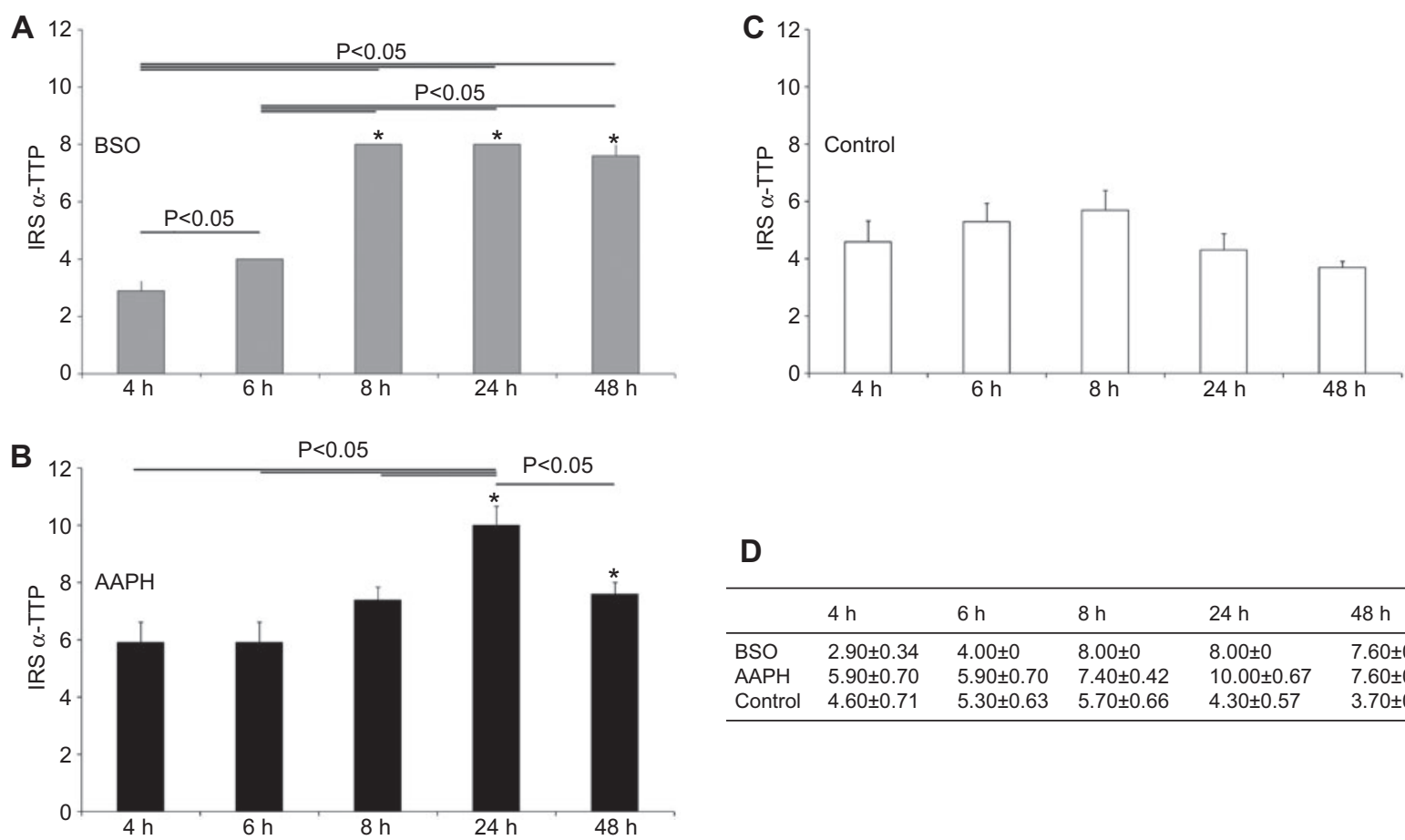

D

\begin{tabular}{llllll}
\hline & $4 \mathrm{~h}$ & $6 \mathrm{~h}$ & $8 \mathrm{~h}$ & $24 \mathrm{~h}$ & $48 \mathrm{~h}$ \\
\hline BSO & $2.90 \pm 0.34$ & $4.00 \pm 0$ & $8.00 \pm 0$ & $8.00 \pm 0$ & $7.60 \pm 0.40$ \\
AAPH & $5.90 \pm 0.70$ & $5.90 \pm 0.70$ & $7.40 \pm 0.42$ & $10.00 \pm 0.67$ & $7.60 \pm 0.40$ \\
Control & $4.60 \pm 0.71$ & $5.30 \pm 0.63$ & $5.70 \pm 0.66$ & $4.30 \pm 0.57$ & $3.70 \pm 0.21$ \\
\hline
\end{tabular}

Figure 2 Time response of $\alpha$-TTP expression in BeWo cells treated with BSO.

(A) $\alpha$-TTP expression is gradually increased, differing significantly from the control after at least $8 \mathrm{~h}$ of treatment. (B) Time response of $\alpha$-TTP expression in BeWo cells treated with AAPH. A $24 \mathrm{~h}$ treatment is essential to reveal a significant difference between treated and untreated cells. (C) $\alpha$-TTP expression of untreated cells. A basal constant $\alpha$-TTP expression is found. (D) Mean IRS \pm SEM for each time-point of the experiment.

in BeWo cells, compared to the corresponding $8 \mathrm{~h}$ AAPH treatment (IRS: $10.00 \pm 0.67$ vs. $7.40 \pm 0.42$, respectively; $\mathrm{P}=0.023$ ) (Figure 2B). Interestingly, after $48 \mathrm{~h}$ of $\mathrm{AAPH}$ treatment, $\alpha$-TTP expression was significantly reduced compared to the $24 \mathrm{~h}$ corresponding treatment (IRS: 7.60 \pm 0.40 vs. $10.00 \pm 0.67$, respectively; $\mathrm{P}=0.037$ ). Further pairwise comparisons between $\alpha$-TTP IRS of AAPH-treated cells vs. untreated cells showed that $\alpha$-TTP is significantly upregulated at least after a 24 -h treatment (IRS: $10.00 \pm 0.67$ vs. $4.30 \pm 0.57$; $\mathrm{P}=0.0001$ ). Despite the significant reduction of $\alpha$-TTP expression after $48 \mathrm{~h}$, the latter remained also significantly above the corresponding $\alpha$-TTP expression of the untreated cells (IRS: $7.60 \pm 0.40$ vs. $3.70 \pm 0.21$, respectively; $\mathrm{P}=0.0005$ ). Thus, it could be concluded that AAPH-induced oxidative stress can also upregulate $\alpha$-TTP expression in BeWo cells, this however being significant after at least a $24-\mathrm{h}$ effect.

\section{Discussion}

As trophoblast (and placenta later on) is in a constant influence of oxidative stress and because the actual placenta can be accessed only after pregnancy has been completed, the need for in vitro models of oxidative stress assessment is imperative. Such models could be used not only for investigating oxidation mechanisms/antioxidants as part of implantation and trophoblast invasion, but also in the case of the above- mentioned pregnancy disorders. In this study, the BeWo cell line has been used. BeWo cells are already an established model in trophoblast research; although derived from human choriocarcinoma, they maintain the ability to differentiate and fuse to syncytiotrophoblast, thus mimicking normal trophoblast [6]. Despite the wide acceptance of BeWo cells in trophoblast research, to our knowledge, this is the first involvement of BeWo in oxidative stress research, especially regarding vitE antioxidant mechanism regulation. The current findings could further support BeWo for further studies at least in the context of investigating vitE antioxidant actions on trophoblast cells. Of note is that another choriocarcinoma cell line has been involved in the field; Jeg3 cells are reported to be involved in oxidation stress induction studies [3, 18, 19].

Animal models have shown that $\alpha$-TTP could play a role in implantation and early trophoblast invasion. $\alpha$-TTP knockout mice present with infertility due to vitE deficiency $[11,17]$. In addition, $\alpha$-TTP has been shown to be increased in implantation sites 3-4 days after implantation in mice [12]. This could imply a role for both $\alpha$-TTP and vitE in normal implantation.

In humans, $\alpha$-TTP has been demonstrated as being presented in term placenta as well as in early-pregnancy trophoblast $[14,24]$. Both cyto- and syncytiotrophoblasts are reported to express $\alpha$-TTP $[14,20]$. Our results are in line with these in vivo findings, as untreated BeWo cells are found to weakly express $\alpha$-TTP. Interestingly, our finding of $\alpha$-TTP 
cytoplasmic reactivity is in concordance with our previous work [14, 24]; nuclear reactivity demonstrated previously by another research group [20] was not identified by our immunocytochemistry. Perhaps, this is because different antibodies were used; in the current study, a well-characterized rat monoclonal anti- $\alpha$-TTP antibody, as described previously [14], was used. On the contrary, nuclear reactivity was revealed by using a polyclonal anti- $\alpha$-TTP antibody [20].

The so found agreement in $\alpha$-TTP staining, between histological samples and BeWo cells, makes BeWo an acceptable model for $\alpha$-TTP studies on trophoblasts.

To this direction, it was shown in this study that chemically induced oxidative stress is associated with an increased $\alpha$-TTP expression in BeWo cells. Such result has not been previously reported in trophoblast cells and could possibly imply a response related to $\alpha$-tocopherol antioxidant action in human trophoblast.

AAPH and BSO induce stress via different mechanisms. AAPH is a water-soluble azo compound that generates free radicals in the form of alkyl radicals spontaneously and continuously at $37^{\circ} \mathrm{C}$ in cell culture medium. These alkyl radicals can react with oxygen to form peroxyl radicals. Peroxyl radicals have been reported to oxidize both cell membranes and intracellular components by initiating lipid peroxidation [2]. BSO is an irreversible inhibitor of $\gamma$-glutamylcysteine synthase and thus of de novo glutathione synthesis [5], drastically reducing cytoplasmic antioxidant capacity. Taking into consideration that at least glutathione is a known placental antioxidant, being affected possibly in any case of oxidative stress, it could potentially be hypothesized that $\alpha$-TTP upregulation could be met not only during normal pregnancy, but also during pregnancy disorders, namely, preeclampsia, GDM, IUGR, and miscarriages. Such finding though needs further in vivo verification. By being verified, this could support further the role of vitE as a placental antioxidant mechanism; modification of dietary vitE intake could then be the next step based on a clinical trial to evaluate the clinical impact of $\alpha$-TTP and vitE on the prognosis of such pregnancy disorders.

Finally, apart from trophoblast research, BeWo cells, being a choriocarcinoma cell line, would also be suitable for investigating the role of oxidative stress in trophoblast disease, as it was reported that trophoblast disease and preeclampsia share a common oxidation stress scheme [7].

In conclusion, we have demonstrated by immunocytochemistry that $\alpha$-TTP can be upregulated, in case of oxidative stress, in BeWo trophoblast cells. This result supports BeWo as a trophoblast cell model for further experiments on $\alpha$-TTP regulation. We speculate that possibly, $\alpha$-TTP is not only involved in normal pregnancy, but also in cases of pregnancy disorders with intense oxidative stress.

\section{References}

[1] Arita M, Nomura K, Arai H, Inoue K. Alpha-tocopherol transfer protein stimulates the secretion of alpha-tocopherol from a cultured liver cell line through a brefeldin A-insensitive pathway. Proc Natl Acad Sci USA. 1997;94:12437-41.
[2] Betigeri S, Thakur A, Raghavan K. Use of 2,2'-azobis(2amidinopropane) dihydrochloride as a reagent tool for evaluation of oxidative stability of drugs. Pharm Res. 2005;22:310-7.

[3] Chen F. Induction of oxidative stress and cytotoxicity by PCB126 in JEG-3 human choriocarcinoma cells. J Environ Sci Health A Tox Hazard Subst Environ Eng. 2010;45:932-7.

[4] Evans HM, Bishop KS. On the existence of a hitherto unrecognized dietary factor essential for reproduction. Science. 1922;56:650-1.

[5] Feugang JM, Donnay I, Mermillod P, Marchandise J, Lequarre AS. Impact of pro-oxidant agents on the morula-blastocyst transition in bovine embryos. Mol Reprod Dev. 2005;71:339-46.

[6] Fischer I, Weber M, Kuhn C, Fitzgerald JS, Schulze S, Friese $\mathrm{K}$, et al. Is galectin-1 a trigger for trophoblast cell fusion?: the MAP-kinase pathway and syncytium formation in trophoblast tumour cells BeWo. Mol Hum Reprod. 2011;17:747-57.

[7] Harma M. Defective placentation and resultant oxidative stress play a similar role in complete hydatidiform mole to that in preeclampsia and early pregnancy loss. Med Hypotheses. 2006;66:100-2.

[8] Hempstock J, Jauniaux E, Greenwold N, Burton GJ. The contribution of placental oxidative stress to early pregnancy failure. Hum Pathol. 2003;34:1265-75.

[9] Hung TH, Skepper JN, Burton GJ. In vitro ischemia-reperfusion injury in term human placenta as a model for oxidative stress in pathological pregnancies. Am J Pathol. 2001;159:1031-43.

[10] Jauniaux E, Watson AL, Hempstock J, Bao YP, Skepper JN, Burton GJ. Onset of maternal arterial blood flow and placental oxidative stress. A possible factor in human early pregnancy failure. Am J Pathol. 2000;157:2111-22.

[11] Jishage K, Arita M, Igarashi K, Iwata T, Watanabe M, Ogawa $\mathrm{M}$, et al. Alpha-tocopherol transfer protein is important for the normal development of placental labyrinthine trophoblasts in mice. J Biol Chem. 2001;276:1669-72.

[12] Kaempf-Rotzoll DE, Igarashi K, Aoki J, Jishage K, Suzuki H, Tamai H, et al. Alpha-tocopherol transfer protein is specifically localized at the implantation site of pregnant mouse uterus. Biol Reprod. 2002;67:599-604.

[13] Kaempf-Rotzoll DE, Traber MG, Arai H. Vitamin E and transfer proteins. Curr Opin Lipidol. 2003;14:249-54.

[14] Kaempf-Rotzoll DE, Horiguchi M, Hashiguchi K, Aoki J, Tamai H, Linderkamp O, et al. Human placental trophoblast cells express alpha-tocopherol transfer protein. Placenta. 2003;24:439-44.

[15] Lappas M, Mitton A, Permezel M. In response to oxidative stress, the expression of inflammatory cytokines and antioxidant enzymes are impaired in placenta, but not adipose tissue, of women with gestational diabetes. J Endocrinol. 2010;204:75-84.

[16] Lash GE, Quenby S, Burton GJ, Nakashima A, Kamat BR, Ray J, et al. Gestational diseases - a workshop report. Placenta. 2008;29(Suppl A):S92-4.

[17] Lim Y, Traber MG. Alpha-tocopherol transfer protein (alphaTTP): insights from alpha-tocopherol transfer protein knockout mice. Nutr Res Pract. 2007;1:247-53.

[18] McAleer MF, Tuan RS. Metallothionein overexpression in human trophoblastic cells protects against cadmium-induced apoptosis. In Vitr Mol Toxicol. 2001;14:25-42.

[19] McAleer MF, Tuan RS. Metallothionein protects against severe oxidative stress-induced apoptosis of human trophoblastic cells. In Vitr Mol Toxicol. 2001;14:219-31.

[20] Muller-Schmehl K, Beninde J, Finckh B, Florian S, Dudenhausen JW, Brigelius-Flohe R, et al. Localization of alphatocopherol transfer protein in trophoblast, fetal capillaries' 
endothelium and amnion epithelium of human term placenta. Free Radic Res. 2004;38:413-20.

[21] Myatt L, Cui X. Oxidative stress in the placenta. Histochem Cell Biol. 2004;122:369-82.

[22] Rani N, Dhingra R, Arya DS, Kalaivani M, Bhatla N, Kumar R. Role of oxidative stress markers and antioxidants in the placenta of preeclamptic patients. J Obstet Gynaecol Res. 2010;36:1189-94.

[23] Remmele W, Stegner HE. Recommendation for uniform definition of an immunoreactive score (IRS) for immunohistochemical estrogen receptor detection (ER-ICA) in breast cancer tissue. Pathologe. 1987;8:138-40.

[24] Rotzoll DE, Scherling R, Etzl R, Stepan H, Horn LC, Poschl JM. Immunohistochemical localization of alpha-tocopherol transfer protein and lipoperoxidation products in human firsttrimester and term placenta. Eur J Obstet Gynecol Reprod Biol. 2008;140:183-91.

[25] Sato Y, Hagiwara K, Arai H, Inoue K. Purification and characterization of the alpha-tocopherol transfer protein from rat liver. FEBS Lett. 1991;288:41-5.
[26] Sato Y, Arai H, Miyata A, Tokita S, Yamamoto K, Tanabe T, et al. Primary structure of alpha-tocopherol transfer protein from rat liver. Homology with cellular retinaldehyde-binding protein. J Biol Chem. 1993;268:17705-10.

[27] Takagi Y, Nikaido T, Toki T, Kita N, Kanai M, Ashida T, et al. Levels of oxidative stress and redox-related molecules in the placenta in preeclampsia and fetal growth restriction. Virchows Arch. 2004;444:49-55.

[28] Wisdom SJ, Wilson R, McKillop JH, Walker JJ. Antioxidant systems in normal pregnancy and in pregnancy-induced hypertension. Am J Obstet Gynecol. 1991;165:1701-4.

The authors stated that there are no conflicts of interest regarding the publication of this article.

Received December 15, 2011. Revised January 17, 2012. Accepted January 23, 2012. Previously published online February 10, 2012. 